Christophe Gremion

IFFP-Lausanne (Suisse)

\section{Accompagner la réflexivité collective, un métier voué à disparaitre?}

Accompanying group reflexivity, a function destined to disappear?

doi: $10.18162 / f p .2019 .490$
Les divers dispositifs d'analyse de pratiques permettent de soutenir une forme de réflexivité collective. Le présent article s'intéresse aux conditions nécessaires pour favoriser de telles démarches. Sur la base de "focus groupes », il montre que le paradigme de l'apprentissage privilégié dans nombre de dispositifs d'accompagnement individuel n'est peut-être pas toujours la posture la plus efficace pour accompagner un collectif. Si une renormalisation est effectuée par les apprenants et que leurs conceptions de l'évaluation et de l'accompagnement sont questionnées, le formateur peut intervenir dans l'exercice d'une évaluationcontrôle, l'étayage réflexif et critique étant dans ce cas délégué au collectif, voire à l'apprenant.

Mots-clés

Réflexivité collective, accompagnement, évaluation

Abstract

The various means of practice analysis serve to support a form of group reflexivity. This paper focuses on the conditions necessary to favour such approaches. Using focus groups, we show that the learning paradigm of many one-on-one support schemes is perhaps not the most effective when supporting a group. If learners go through a renormalization process and their conceptions of the evaluation and support are questioned, the trainer can play his or her role in the control evaluation, since, in this case, the reflexive and critical support is delegated to the group, and even to the learner.

Keywords Group reflexivity, accompaniment, assessment.
Le développement de la posture réflexive des enseignants ${ }^{1}$ intéresse de nombreux formateurs et chercheurs. Considérée parfois comme un objet ou un but de formation, elle peut aussi être vue comme un outil, un moyen de se former. Ces deux intentions ne sont pas contradictoires et œuvrent au service de la professionnalisation des personnes. En effet, avoir une posture réflexive - si celle-ci n'est pas synonyme de rupture avec les savoirs théoriques (Beauchamp, 2012; Tardif, 2012) - impose d'analyser sa pratique, de se rendre compte de ses forces ou faiblesses, de prendre des décisions dans des situations nouvelles, de mettre en évidence des besoins de formation et, in fine, d'être plus efficace et pertinent dans son activité, ce qui correspond au but du mouvement de professionnalisation-formation (Wittorski, 2014) de la formation à l'enseignement.

L'exercice de la posture réflexive, et l'autoévaluation qu'elle induit, permet d'une part de guider son action en la comparant à des référents et des prescrits du métier afin d'entrer dans le genre professionnel, mais elle offre aussi la possibilité d'adopter une posture critique face à ces mêmes référents et prescrits, éléments émancipateurs permettant de trouver son propre style professionnel (Clot, 1999; Jorro, 2016). S'autoévaluer, ou analyser sa propre pratique, est un exercice complexe qui ne se fait que difficilement seul, raison pour laquelle de nombreuses formations d'enseignants mettent en place un dispositif d'accompagnement ou de médiation (Le Boterf, 2015, p. 147). L'autoévaluation devient alors socialisée et l'exercice, s'il est ainsi soutenu, n'en demeure pas moins complexe tant les stratégies mises en œuvre par les personnes en formation sont nombreuses (Saussez et Allal, 2007).

L'authenticité de l'autoévaluation s'invite alors dans la discussion. Tout d'abord, la question est de savoir si la personne s'engage d'ellemême dans une démarche autoévaluative ou si elle le fait sous contrainte ressentie, ce qui pourrait l'amener à simuler, à inventer, à 
théâtraliser (Gremion,2017). L'accompagnateur joue un rôle important dans cette démarche également. Est-il perçu comme représentant d'une norme donnée, attendue par l'institution, ou au contraire est-il celui qui permet de remettre cette norme en débat (Vial et Caparros-Mencacci, 2007) en offrant les conditions nécessaires à un tel engagement : la bienveillance ou l'absence de jugement pour ne citer que ces deux dimensions souvent évoquées?

La question est importante, car c'est bien dans une intention de professionnalisation que sont offertes ces formations, ce qui implique de développer l'autonomie au service de prises de décisions responsables dans des situations inattendues (Lemosse, 1989). La dimension de responsabilité ne renvoie dans ce cas plus au genre professionnel ni au style professionnel, mais bien à l'éthos professionnel dont parle Jorro (2016), cette posture qui implique parfois de remettre en question voire de transgresser certaines normes (Alhadeff-Jones, 2014, p. 10) pour s'assurer d'une action éthique et altruiste.

Cet éthos professionnel se construit dans une dialectique qui implique la réflexivité sociale et la réflexivité individuelle (Kaufmann, 2001), le tout mis en perspective avec les valeurs éthiques de la profession. Ce travail est à la base des codes de déontologie existants pour certaines professions. Ces codes, qui n'existent pas à large échelle pour la profession enseignante, sont la mise en visibilité des intentions collectives et partagées des professionnels de la branche.

Accompagner la personne en formation professionnalisante revient ainsi à la placer dans une situation paradoxale: "Sois autonome, mais contribue volontairement à la normalisation de ton action " (Maulini et Vincent, 2014). Ce dilemme a souvent été mis en évidence dans les études s'intéressant à l'accompagnement (Boucenna, 2017; Boucenna, Charlier, Perréard-Vité et Wittorski, 2018; Donnay et Charlier, 2008; Gremion et Maubant, 2017; Vialle, 2005). Si l'accompagnateur adopte une posture prescriptive, la co-évaluation devient une demande d'autocontrôle, un jeu qui n'est pas sans rappeler l'hypothétique « ajustement réciproque des postures des enseignants et des élèves » (Bucheton et Soulé, 2009, p. 42). L'accompagné cherche à correspondre à un genre ou à un style professionnel. Dans ce cas, sa réflexivité s'exercera par confirmation (Boltanski,2009,p. 152) et sera assujettie à la réflexivité sociale. La personne en formation exécute, applique, fait son métier d'élève, ne développant ni son autonomie ni sa responsabilisation face à ses actions. À l'inverse, en choisissant une posture interprétative, la coévaluation devient une mise en débat des décisions et des valeurs, ce qui permet le questionnement de sa pratique, la recherche de son style professionnel. La réflexivité s'opère par analyse et critique selon ses propres valeurs. La personne en formation s'émancipe, mais au risque de ne plus correspondre au genre professionnel, ce qui met l'institution, dans son rôle certificateur, en situation délicate.

Nous le voyons, s'autoévaluer ne se fait que difficilement seul et, lorsque cette pratique est accompagnée, soit le point de vue de l'un prime sur celui de l'autre - ce qui pousse la personne en formation à rechercher la normalisation de son action dans une logique applicationniste -, soit les deux points de vue sont mis en débat dans une intention émancipatrice au risque de ne plus permettre cette nécessaire normalisation de l'action. Comment la réflexivité collective entre personnes en formation peut-elle proposer une issue positive à ce dilemme?

En augmentant le nombre de points de vue dans l'échange réflexif, les idées ne peuvent plus être prises comme des recettes à appliquer puisqu'elles imposent de faire des choix réfléchis plutôt que d'accepter une forme de doctrine, d'exercer cette « lucidité qui [...] donne la force de mettre en cause les vérités institutionnellement confirmées et les vérités de sens commun » (Boltanski, 2009, p. 152). 
De plus, les opinions exprimées entre pairs nétant pas des avis émis par un professionnel reconnu comme expert, chacun est là aussi amené à faire des choix, à prendre des décisions par lui-même, ce qui est déterminant dans un dispositif à visée professionnalisante. C'est ce que propose d'observer cette recherche exploratoire.

L'analyse des propos de 37 personnes, impliquées dans des dispositifs favorisant le travail d'analyse de pratiques entre pairs, a permis de mieux comprendre l'apparition de cette réflexivité collective. Elle montre que lorsque le groupe prend en charge ce travail d'enquête et de renormalisation, il semble ne plus avoir besoin d'accompagnement extérieur et il attend des formateurs qu'ils reprennent un rôle d'expert ou de garant externe.

\section{Cadre théorique}

Entrer dans une nouvelle profession est un travail de construction de soi complexe. Le carré dialectique proposé par Kaufmann (2001) offre une lecture intéressante de cette complexité. L'auteur définit la socialisation de l'individu comme une discussion entre quatre pôles : l'habitude, le cadre de socialisation, la réflexivité individuelle et la réflexivité sociale.

L'habitude est constituée des schèmes d'action mobilisés par la personne dans différentes situations. Elle n'est pas purement répétitive et s'adapte selon les contradictions identifiées. Ces contradictions sont le fruit de cette dialectique : entre l'habitude (ce que je fais) et la réflexivité collective (ce que je pense que devrait faire un professionnel dans cette situation) d'abord. La comparaison entre cet observable et un attendu social intériorisé contribue à la construction du genre professionnel (Clot, 1999). Le cadre de socialisation (lieu dans lequel se déroule l'action) influe également sur la représentation de la réflexivité sociale et peut faire apparaître de nouvelles contradictions entre ces trois pôles. Ces divergences construisent petit à petit la réflexivité individuelle (ce que je pense que je dois faire) constitutive du style professionnel.

Les tensions potentielles entre ces deux réflexivités sont au cœur de cette recherche. Si, systématiquement, la personne règle la contradiction en s'appuyant sur la norme sociale, elle applique une manière de faire sans engagement personnel, effet opposé aux intentions de professionnalisation. Cette situation se retrouverait dans une organisation de travail à prescriptions strictes comme une organisation taylorienne par exemple (Le Boterf, 2015, p. 171). Ce n'est pas le cas de la formation à l'enseignement. À l'inverse, si elle ne tient pas compte de la norme sociale et n'agit que selon ses propres valeurs, le risque de ne plus répondre aux attentes du genre professionnel augmente, ce qui n'est pas socialement souhaitable. De la dialectique entre ces deux réflexivités apparaît un nouvel équilibre que nous pouvons associer à l'éthos professionnel (Jorro, 2016), capacité de l'individu à prendre du recul sur son action et sur ses prises de décisions, en tenant compte des normes sociales, de ses valeurs personnelles et du sens éthique.

Boltanski (2009, p. 152-155) nous propose une autre distinction entre deux types de réflexivités, très complémentaires à celles proposées par Kaufmann. Dans son précis de sociologie de l'émancipation, il nous invite à analyser la réflexivité par la critique en regard de la réflexivité par la confirmation.

Pour adopter une posture critique, la personne doit tout d'abord s'engager personnellement en faisant preuve de lucidité. Ce sont les conditions qui lui donnent la force de remettre en cause les «vérités institutionnellement confirmées et les vérités de sens commun» (p.152). Cette énergie à déployer 
pour gagner en autonomie n'est pas sans rappeler le concept d'« empowerment " nécessaire pour engager un processus de changement (Gather Thurler, 2000), qu'il soit individuel ou collectif. Lorsque la réflexivité par confirmation s'invite, par contre, l'acteur n'est pas actif dans le processus réflexif, puisque celui-ci est porté par l'institution. Il devient l'exécutant, le « desservant de l'être sans corps de l'institution "(Boltanski, 2009, p. 154). La prise de risque n'existe pas tant qu'il accomplit ce que l'on attend de lui. Dans le contexte de formation professionnalisante, la critique semble ainsi plus efficace que la confirmation. Mais l'une ne va pas sans l'autre. La critique, bien qu'elle puisse bousculer l'ordre établi, s'appuie justement sur cet ordre déjà en place pour proposer des alternatives. Sans normes collectivement reconnues, la critique n'aurait simplement pas lieu d'exister.

Lors du travail de renormalisation (Schwartz, 2004, p. 19), d'appropriation de la norme par la personne en formation, ces deux formes de réflexivités se complètent, se répondent. Dans le cas de réflexivité collective, le point de vue des pairs peut être objet de critique, et ce, d'autant plus facilement qu'il n'a pas la valeur d'un ordre immuable établi. Il en va tout autrement du point de vue du formateur.

Avoir une posture réflexive face à sa pratique impose un acte d'autoévaluation. Mais quelles représentations a la personne en formation de cette notion d'évaluation? Les réponses seraient nombreuses et en faire un recensement exhaustif serait utopique! Il paraît par contre assez certain que de nouvelles dialectiques pourraient être identifiées : une évaluation qui sert au contrôle ou à tout le reste (Vial, 2012), une évaluation à visée interne ou externe, une évaluation pour développer ou pour normaliser, une évaluation pour comprendre ou pour prouver (Berthiaume, Lanarès, Jacqmot, Winer et Rochat, 2011), une évaluation par confirmation ou par critique (Boltanski, 2009), une évaluation quantitative ou qualitative (Jorro, 2016)... Toutes ces dimensions de l'évaluation vont avoir de l'influence sur les formes autoévaluatives pratiquées par la personne en formation (Jorro, 2004).

Mais cette autoévaluation est socialisée (Jorro et Van Nieuwenhoven, 2019; Saussez et Allal, 2007), nous l'avons vu, et le rôle que doit jouer le formateur dans le dispositif influe également fortement sur la posture autoévaluative de l'apprenant (Gremion et Coen, 2016). S'il sent, ou sait, qu'il doit rendre des comptes pour prouver sa conformité à une attente précise, il ne sera que peu enclin à mettre en évidence sa dernière erreur pour tenter de comprendre quel apprentissage celle-ci lui suggère pour ne citer que cet exemple simple.

Dans le cas de la réflexivité collective, l'évaluation mutuelle (entre pairs), sans le regard du formateur, devrait permettre de réduire au maximum les effets indésirables de cette autoévaluation socialisée, les regards des pairs n'ayant pas cette valeur «d'ordre établi ». Ainsi, la personne en formation définit, à l'aide de ses pairs, l'attendu qui serait souhaitable pour elle. Dès lors, elle peut mener un travail d'enquête pour comprendre ce qui se passe dans sa pratique ou un travail de comparaison entre un attendu (ou espéré par elle-même et par ses pairs) et un constat (ou perçu). Autoévaluation et autocontrôle redeviennent ainsi des gestes éthiques au service du formé et du plus grand nombre de personnes (Hadji, 2012, p. 290).

Le dialogue qui s'instaure entre la réflexivité individuelle de chaque membre d'un groupe et la représentation que chacun a de la réflexivité sociale propre au métier est un puissant facteur de renormalisation(Schwartz,2004). Elle estentendue ici comme un « travailpermanent de renouvellement des règles d'élaboration de l'expérience et des règles à suivre dans l'action » (Lussi Borer et Muller, 2014, p. 130) et est associée à une étape de référentialisation (Figari, 1994) de l'apprentissage visé par la personne en formation elle-même. Cette action est le constituant central de l'autoévaluation afin de 
permettre la dialectique entre recherche de conformité et regard critique. La moindre place prise par le formateur dans les phases de renormalisation et d'autoévaluation pourrait être une piste de réponse à notre question de départ, à savoir : comment la réflexivité collective entre personnes en formation peutelle proposer une issue positive au dilemme qui oppose normalisation volontaire de son action et autonomie attendue?

\section{Méthodologie}

Pour le savoir, nous nous sommes intéressés à deux cours donnés à l'Institut fédéral des hautes études en formation professionnelle (IFFP) par trois formateurs. Bien que portant sur des sujets différents, les deux dispositifs s'organisent selon la même ingénierie pédagogique. Dans chaque classe, les étudiants sont répartis en groupes de trois ou quatre personnes pour une série de tâches d'analyse de pratiques devant permettre la professionnalisation (Boucenna et al., 2018, p. 185) et favoriser l'apprendre en situation (Maubant, 2013), et qui peuvent être synthétisées ainsi :

- s'approprier une série de concepts théoriques;

- planifier une activité dans sa classe;

- la présenter à ses collègues du groupe pour en discuter, pour la confronter aux concepts théoriques vus préalablement;

- améliorer la préparation de l'activité;

- élaborer en groupe une grille d'évaluation des activités réalisées en classe;

- réaliser l'activité dans sa classe en se filmant;

- pratiquer une autoévaluation puis une évaluation mutuelle des séquences filmées à l'aide de la grille élaborée;

- élaborer une nouvelle version de l'activité en tenant compte des avis, des pistes, des éléments théoriques mis en évidence;

- mettre en évidence les apprentissages réalisés durant cette démarche.

En fin d'année, les participants $(n=60)$ ont été invités à participer à cette recherche exploratoire. Les volontaires $(n=37)$ ont pu choisir de nous accorder du temps pour des entretiens individuels $(n=15)$ ou pour des « focus groupes » (8 rencontres pour 22 répondants au total).

Les entretiens semi-directifs, de 40 à 75 minutes, ont été menés par un formateur de l'institution n'intervenant pas dans ces cours. Les thèmes abordés ont traité des différentes modalités d'évaluation proposées, des moments propices à l'appropriation de la norme, du rôle que jouent les Autres dans le processus réflexif. D'autres informations, centrées sur l'utilisation d'un ePortfolio, ont été recueillies pour une recherche menée en parallèle (Vaudan Méresse et Gremion, 2018).

Six « focus groupes » $(n=16)$ ont permis d'aborder explicitement la question de la place du collectif et celle du formateur dans l'apparition de la réflexivité collective. Les échanges, chaque fois qu'ils abordaient cette question, ont été retranscrits et soumis à une analyse par catégories issues de notre cadre théorique. Parmi celles-ci, citons :

- Fonctionnement du groupe;

- Activité d'évaluation entre pairs;

- Évaluation interne (personne) vs externe; 
- Évaluation interne (vue cette fois depuis le groupe) vs externe;

- Évaluation se rapportant à un modèle (prescriptions strictes) ou à une mise en débat des prescrits ouverts;

- Représentant de l'expertise (individu, collectif ou formateur);

- Conditions pour permettre la remise en question dans l'autoévaluation;

- Perception du rôle du formateur;

- Sources d'apprentissages identifiées.

\section{Résultats}

Dans les résultats, nous vous présentons dans un premier temps les six groupes pour cette recherche exploratoire (Tableau 1). Pour chacun, nous résumons brièvement la situation vécue dans le collectif et mettons en évidence les rôles tenus par le formateur $(F)$ et par le groupe $(G)$. Ensuite, nous analyserons quelques verbatims qui nous semblent utiles à la compréhension du fonctionnement de chaque groupe.

\section{Tableau 1}

Descriptif des six groupes.

\begin{tabular}{|c|c|c|c|c|}
\hline & Situation rapportée par le groupe & Rôle des uns et des autres dans le groupe & 을 & 莺 \\
\hline grF & $\begin{array}{l}\text { Seul le formateur est légitime dans son rôle d'expert. S'il ne valide pas, } \\
\text { la situation est trop déstabilisante. Entre pairs, se dire ce qui va ou ne va } \\
\text { pas semble très difficile. }\end{array}$ & $\begin{array}{l}\text { L'évaluation, formative ou certificative, semble vue } \\
\text { comme un jugement peu acceptable entre pairs. Les } \\
\text { rôles sont laissés au formateur. }\end{array}$ & $\mathrm{F}$ & $\mathrm{F}$ \\
\hline $\operatorname{grC}$ & $\begin{array}{l}\text { Seul le formateur est légitimé dans le rôle d'expert. Le groupe ne } \\
\text { fonctionne pas très bien et les apports du groupe sont appréciés de } \\
\text { manières très différentes selon les étudiants. }\end{array}$ & $\begin{array}{l}\text { Tous les rôles sont attendus du formateur et le groupe } \\
\text { n'existe que peu en tant qu'unité. La posture de chacun } \\
\text { est de type applicationniste. }\end{array}$ & & \\
\hline grD & $\begin{array}{l}\text { Le prof, c'est l'expert : on a besoin de son retour. Le dispositif propose de } \\
\text { la réflexivité collective, mais notre habitus d'étudiant rend la situation } \\
\text { inconfortable. Côté organisationnel, il est aussi plus simple de venir au } \\
\text { cours lorsqu'on nous l'impose que de trouver un temps pour travailler en } \\
\text { sous-groupe, ce qui est vu comme source de tensions. }\end{array}$ & $\begin{array}{l}\text { Tous les rôles sont attendus du formateur et le groupe } \\
\text { n'existe que peu en tant qu'unité. Au niveau individuel, } \\
\text { l'autoévaluation sur la base des traces vidéo est vue } \\
\text { comme positive. }\end{array}$ & $\mathrm{F}$ & $\mathrm{F}$ \\
\hline grA & $\begin{array}{l}\text { Le groupe sent que l'expertise se déplace, représentée au départ par le } \\
\text { formateur, elle devient l'affaire du groupe. Les membres relèvent que } \\
\text { la remise en question est plus facile devant ses pairs, mais regrettent } \\
\text { l'absence de validation de la part du formateur. }\end{array}$ & $\begin{array}{l}\text { Le groupe s'accepte comme expert-critique et attend du } \\
\text { formateur quili valide, confirme. }\end{array}$ & G & $\mathrm{F}$ \\
\hline grB & $\begin{array}{l}\text { Le groupe aimerait les retours du formateur, mais celui-ci refuse ce rôle. } \\
\text { La situation déstabilise les étudiants dans un premier temps, puis le } \\
\text { groupe entre dans le travail d'évaluation mutuelle et juge la démarche } \\
\text { formatrice. }\end{array}$ & $\begin{array}{l}\text { Le groupe s'accepte comme expert et évaluateur. II } \\
\text { attend du formateur qu'il organise, qu'il rythme. }\end{array}$ & G & G \\
\hline grE & $\begin{array}{l}\text { La norme est représentée par les membres du groupe, qui devient } \\
\text { « expert » aux yeux des participants. Le formateur n'est que } \\
\text { «l'organisateur de la séquence didactique ». }\end{array}$ & $\begin{array}{l}\text { Le groupe s'accepte comme expert et évaluateur. II } \\
\text { attend du formateur qu'il organise, qu'il rythme. }\end{array}$ & G & G \\
\hline
\end{tabular}


La première colonne donne le numéro du groupe afin de pouvoir ensuite y rattacher les verbatims qui seront présentés. Les deux colonnes de fin précisent qui ( $\mathrm{G}$ ou F), dans le dispositif, a pris en charge la dimension d'évaluation par conformité vs par critique. Nous remarquons ainsi que 3 groupes sur 6 n'ont pris la responsabilité d'aucune part de l'évaluation. Deux d'entre eux semblent avoir eu de la peine à se constituer en entité propre ( $\mathrm{grC}$ et $\mathrm{grD})$ alors que, dans le troisième $(\mathrm{grF})$, les membres semblent ne pas se permettre d'exprimer un avis (ou un jugement selon leur propos) sur le travail de leurs collègues.

Les trois autres groupes ( $\mathrm{gr} A, \operatorname{grB}$ et $\mathrm{grE}$ ) prennent en charge la partie réflexive critique de l'évaluation et en parlent comme d'une démarche formatrice permettant les remises en question. Dans le groupe A, le formateur est vu comme « la personne qui valide ", côté contrôle ou certificatif de l'évaluation. Les deux autres groupes quant à eux se considèrent comme responsables des deux dimensions de l'évaluation et ne voient dans le formateur que le rôle d'organisateur du dispositif de formation.

En observant plus précisément les propos des répondants, le premier élément qu'ils mettent en évidence est la richesse des apports du groupe de pairs. Que ce soit lors du visionnage des vidéos de chacun ou lors des séances de préparation de la grille d'évaluation, ces activités sont considérées comme source importante d'apprentissages et de prises de conscience.

"Le partage dans le groupe, c'est là quion a le plus appris » (grC 5’15).

"La vidéo, c'est ce qui m’a permis de prendre conscience de certaines choses dans mon enseignement. » (grD 11'50).

Mais comme nous le relevions précédemment, les conditions de fonctionnement du groupe permettent (ou non) cette réflexivité collective. Ainsi, dans certains groupes, l'activité d'évaluation mutuelle, que les répondants nomment facilement «faire des commentaires » de par l'ingénierie proposée, a eu de la peine à fonctionner. Parfois par manque d'engagement, "dans mon groupe, il n'y a pas vraiment eu des commentaires des autres" (grC 1'00), d'autres fois simplement parce que trouver du temps pour travailler ensemble, hors du temps de cours et du temps de travail, n'est pas si simple: "Mais c'est vraiment important que le groupe fonctionne bien [...] parce que, pour nous, il était parfois difficile de se voir» (grD 22'10).

Lorsque le travail peut se réaliser comme prévu, les regards croisés entre pairs ont été positifs : «Entre nous, on hésitait, on cherchait un regard avec une forme d'expertise. Le retour des autres, avec leurs "lunettes", nous l'a apporté. Et pour moi, cétait important ce regard des autres... Moi, jeetais plus sévère avec moimême! » (grE 8'47).

Ce verbatim nous fait sentir l'inconfort que représente, au début, le fait de devoir pratiquer une autoévaluation et une évaluation sans le regard ou le retour du formateur, sans le point de vue de cette réflexivité sociale légitimée. Il met également en évidence les difficultés que génère l'autoévaluation stricto sensu. "J'étais plus sévère », ou, ci-dessous, "je n’y arrive pas ».

"Moi, seul, je n'arrive pas. J'ai besoin de l'avis de quelqu'un, je n'arrive pas à m'autoérvaluer, j'ai besoin que quelqu'un me le dise " (grA 21'20).

"Il y a quelque chose qui se joue entre nous trois, avec plus de positif que de critique. Ça doit être difficile de dire à un collègue que ça ne va pas » (grF 15'15). 
Dans les groupes qui ont bien fonctionné, cette dimension, la difficulté de dire « que ça ne va pas » dans le travail de l'autre, se retrouve chez plusieurs répondants, principalement pour deux raisons : certains estiment qu'ils devraient être experts pour se permettre de donner leur avis, d'autres ont un avis assez clair, mais préfèrent ne pas le donner de peur que l'évaluation ainsi produite influe sur la réussite ou non du module de leurs collègues, que l'évaluation soit tout de même, en partie, élément de contrôle et de certification.

Dans ces dispositifs, les formateurs souhaitent accompagner le processus, lâcher du contrôle pour permettre plus d'autonomie et de responsabilisation. Les étudiants ne sont pas toujours à l'aise avec cette posture, l'élément n'est pas nouveau : "On aurait aimé qu'il valide. À la place de cela, il nous a fait confiance et a cheminé avec nous. C'est plus (+) de l'accompagnement » (grB 3’20).

Les données recueillies mettent à plusieurs reprises cet élément en évidence. Le formateur est celui qui sait et qui permet la réflexivité par confirmation, alors que le groupe, constitué de personnes qui se considèrent toutes comme débutantes, permet la réflexivité par la critique. Cette répartition des rôles est attendue par la majorité des répondants.

«Pour nous, le formateur, c'est l'autorité. C'est lui qui a les jetons, les projets» (grB 33’00).

"Mais on a vraiment besoin du retour du formateur pour se sentir en confiance. Sans cela, on ne sait pas trop si on est dans le juste, ce n'est pas très confortable » ( $\mathrm{grC} \mathrm{8’30).}$

Nous le voyons bien dans ces deux extraits, les groupes légitiment le rôle de contrôle du formateur, l'appellent même. Cette situation pourrait paraître contre-productrice dans un accompagnement individuel, le point de vue du formateur devenant la norme que l'on ne remet pas en question. Mais ici, la situation change, la répartition des rôles est différente et, si le groupe prend en charge la dimension interprétative, il délègue à l'enseignant celle de contrôle. Les deux fonctions de l'évaluation sont présentes, les deux formes de réflexivités (confirmation et critique) aussi. Mais est-ce le fruit d'un choix de posture de la part des étudiants? Cet extrait nous fait plus penser à une répartition habituelle dans le monde scolaire :

"On nous dit souvent qu'il n'y a pas de juste et pas de faux, mais on arrive diffcilement à se positionner. On a besoin d'être rassurés. On a peut-être été trop habitués à ça aussi » (grD 9'40).

Enfin, que ce soit par habitude ou par choix, il semblerait bien qu'une progression soit appelée par les répondants. Dans un premier temps, la réflexivité par confirmation semble nécessaire afin de favoriser la confiance en son propre jugement.

«Oui, le prof, c'est l'expert! Il donne une ligne claire de ce qui est attendu » (gr 10’05).

Cette confiance établie apparaît comme un prérequis nécessaire pour accepter d'entrer dans un processus de réflexivité par critique face à sa pratique, face à celle de ses collègues ensuite.

"Au départ, je donnais plus de poids aux commentaires du prof. Ensuite, ça s’ést équilibré" (grA 7’05). 
Lorsque ces rôles semblent bien répartis, que cet équilibre est trouvé et que la confiance est établie, le poids que les étudiants attribuent au regard du formateur diminue. L'hypothèse de départ, l'idée du dilemme qui oppose normalisation volontaire de son action et autonomie attendue semble trouver un épilogue heureux :

"L'évaluation mutuelle est extrêmement formatrice. Si c'est le formateur qui évalue, tu te décharges. Mais là, tout d'un coup, tu es porteur de cette évaluation » (grB 29'10).

Mais si le formateur n'évalue plus, si sa présence diminue, c'est alors la dimension de réflexivité par confirmation face au genre professionnel qu'il incarne qui perd de son importance, créant un nouveau déséquilibre :

«La force du groupe, c'est qu'il permet la remise en question [...], mais là, on n'avait plus l'instance supérieure, le formateur, qui venait nous dire... » (grA 6’00).

Ces propos sont issus d'une classe dans laquelle le formateur suivait de manière moins régulière le travail de ses étudiants. L'absence relative de remarques, de feedbacks dans les dossiers des étudiants a été vécue par certains comme peu rassurante :

«Mais les remarques du prof sont importantes pour nous rassurer, on attendait que le formateur valide » (grE 8'47).

Et la validation première que les étudiants attendent semble bien être le jugement de valeur, la reconnaissance de la légitimité face au genre professionnel.

«Mais qui je suis pour me dire que je suis une bonne enseignante ou bien pas? (grB 39'20).

"C'est le rôle à donner au prof. Il est plus légitime, il n'a pas le même rôle que nous" (grF 16’30).

\section{Vers quelques éléments conclusifs}

Les éléments apportés par les répondants nous montrent que même lorsque le formateur adopte une posture interprétative propre à la réflexivité par critique, il incarne la norme et implique une réflexivité par confirmation aux yeux des personnes en formation. Il représente ainsi une réflexivité sociale (Kaufmann, 2001) qui a plus de poids que la réflexivité individuelle propre à chaque étudiant. Selon les travaux de Vial (2006), cette association de la norme avec le formateur semble normale, c'est la raison pour laquelle l'auteur insiste bien sur la différence à faire entre former et accompagner. I1 serait intéressant de voir s'il en est de même dans la relation accompagné-accompagnateur. Dans la majorité des instituts de formation d'enseignants, l'accompagnement, lorsqu'un tel dispositif est mis en place, est assuré par des formateurs de l'institution. Ceux-ci tiennent des rôles différents dans ces deux situations, mais cette dissociation des fonctions est-elle toujours très claire pour les personnes en formation? Une précédente recherche nous a montré que, pour la différenciation entre les rôles de l'évaluateur et de l'accompagnateur, la distinction nétait pas si simple (Gremion, 2017). 
Toujours au niveau de la posture adoptée par le formateur, les répondants mettent en évidence un autre élément qui mérite notre attention : lorsque le formateur souhaite rester dans une posture purement interprétative, il ne valide pas, il questionne. Dans le même temps, si le groupe d'étudiants prend en charge cette réflexivité collective, le travail interprétatif se fait entre eux, sans le regard du formateur. Ils attendent de sa part non pas de nouvelles questions, mais une validation :1) de la qualité de leurs séquences d'enseignement ou 2) de la pertinence de leur posture interprétative à l'intérieur du groupe. Mais si ce dernier manque de retours de la part du formateur, la machine semble s'enrayer et perdre de son efficacité.

Pour que la réflexivité collective se mette en place dans ce petit groupe, il semble nécessaire que l'évaluation soit perçue, durant la formation, comme un outil au service du développement formatif et non au service du contrôle. Lorsque le développement représente le but du travail, la critique - cette recherche de nouvelles pistes, de solutions ou de compréhension - constitue l'activité principale du groupe. Cette autonomie dans le travail d'enquête semble s'appuyer sur des retours plus ou moins réguliers du formateur, retours plutôt prescriptifs puisqu'il devient, dans ce cas, garant de la démarche aux dires des étudiants, garant d'un retour par confirmation. Par contre, lorsque le contrôle orchestre ce travail, la recherche de conformité constitue l'activité centrale du groupe. Mais dans ce cas, le formateur ne devient pas pour autant le garant de la critique. Le groupe reste dans une attente de retours au service de la validation. L'absence de critique empêche le travail de renormalisation, les étudiants ne s'approprient pas une norme qui représenterait à leurs yeux ce que signifie « un enseignement efficace ». Pour eux, la norme est portée soit par l'institution (il faut faire une leçon qui soit compatible avec les attentes de l'IFFP), soit par le formateur. Un travail de déconstruction des représentations quant aux rôles de l'évaluation semble donc nécessaire pour permettre l'engagement dans la démarche de ces petits collectifs.

Ce constat interpelle. Alors que, dans les dispositifs cherchant à développer une réflexivité individuelle, la posture de l'accompagnement non guidant (Vial, 2006) ou du compagnonnage réflexif (Donnay et Charlier, 2008) est reconnue comme des plus efficaces au service de l'engagement de la personne dans son processus de formation, il semble que la situation soit différente dans le cas de la réflexivité collective. En effet, si les autres membres du groupe permettent la réflexivité par critique, le formateur est attendu comme levier de la réflexivité par confirmation, et perd petit à petit son rôle d'accompagnateur. Si le paradigme de l'apprentissage se retrouve au centre des dispositifs d'accompagnement individuel, c'est celui de l'enseignement qui paraît reprendre sa place dans l'accompagnement collectif.

Un changement de paradigme au service d'une réflexivité collective qui nécessite trois conditionscadres selon nous.

Premièrement, ce changement n'est possible que si un travail de référentialisation et de renormalisation a été effectué par le collectif. Le but de cette étape est de ne plus laisser entendre que la norme est un attendu de l'institution ou du formateur, mais bien un attendu pour le bon fonctionnement de la société, au service du plus grand nombre (Hadji, 2012, p. 290), et que la personne décide de " porter elle-même » en tant qu'acteur principal de sa propre formation. 
Ensuite, les conceptions des finalités de l'évaluation et de l'autoévaluation doivent être remises en question. Comme outil au service du développement et de la reconnaissance de la personne, l'évaluation devient le gouvernail nécessaire à cet acteur principal, lors de son autoévaluation, voire de son autocontrôle. En effet, lorsqu'il consiste à permettre à la personne en formation de comparer son action avec son propre attendu, avec sa propre norme, le contrôle prend un tout autre visage.

Enfin, la renormalisation et l'autoévaluation s'effectuent efficacement lorsqu'elles sont le travail d'un collectif de pairs. Dans cette élaboration de norme, il semble que la symétrie de la relation soit nécessaire pour permettre la mise en discussion et la critique d'une norme qui n'est pas la sienne.

Lorsque ces conditions sont réunies, la dimension d'accompagnement est assurée par le groupe luimême et le formateur s'en trouve libéré. Il peut ainsi retrouver sa fonction de formateur et d'expert, sans soumettre ses étudiants à des injonctions paradoxales.

Dans ce cas, le vrai accompagnement pourrait-il être de fournir aux personnes les moyens de s'accompagner elles-mêmes? Et en serait-il de même dans l'accompagnement individuel?

\section{Note}

1 Le masculin, utilisé lors de l'écriture, renvoie naturellement à un collectif mixte, et ce, tout au long du texte.

\section{Références}

Alhadeff-Jones, M. (2014). Pour une approche réflexive et critique des rapports entre temporalités et professionnalisation. Phronesis, 3(4), 4-12. http://dx.doi.org/10.7202/1028781ar

Beauchamp, C. (2012). Un cadre conceptuel pour mieux comprendre la littérature sur la réflexion en enseignement. Dans M. Tardif, C. Borgès et A. Malo (dir.), Le virage réflexif en éducation : où en sommes-nous 30 ans après Schön? (p. 21-45). Bruxelles : De Boeck. http://dx.doi.org/10.3917/dbu.tardi.2012.01.0021

Berthiaume, D., Lanarès, J., Jacqmot, C., Winer, L., \& Rochat, J.-M. (2011). L'évaluation des enseignements par les étudiants (EEE). Une stratégie de soutien au développement pédagogique des enseignants ? Recherche et formation, (67), 53-72. https://doi.org/10.4000/rechercheformation.1387

Boltanski, L. (2009). De la critique : Précis de sociologie de l'émancipation. Paris : Gallimard.

Boucenna, S. (2017). L'accompagnement : symétrie dans les asymétries? Phronesis, 6(4), 60-70. http://dx.doi.org/10.7202/1043981ar

Boucenna, S., Charlier, É., Perréard-Vité, A. et Wittorski, R. (2018). L'accompagnement et l'analyse des pratiques professionnelles : des vecteurs de professionnalisation. Octarès.

Bucheton, D. et Soulé, Y. (2009). Les gestes professionnels et le jeu des postures de l'enseignant dans la classe : un multiagenda de préoccupations enchâssées. Éducation et didactique, 3(3), 29-48. http://dx.doi.org/10.4000/educationdidactique.543

Clot, Y. (1999). La fonction psychologique du travail. Paris : Presses universitaires de France.

Donnay, J. et Charlier, E. (2008). Apprendre par l'analyse de pratiques : Initiation au compagnonnage réflexif( $2^{\mathrm{e}}$ éd.). Namur : Presses universitaires de Namur.

Figari, G. (1994). Évaluer : quel référentiel? Bruxelles : De Boeck. 
Gather Thurler, M. (2000). Innover au cœur de l'établissement scolaire. Paris : ESF Éditeur.

Gremion, C. (2017). Place de l'accompagnement et du contrôle dans les dispositifs de formation en alternance. Phronesis, 6(4), 99-113. http://dx.doi.org/10.7202/1043984ar

Gremion, C. et Coen, P.-F. (2016). De l'influence du contrôle dans les dispositifs d'accompagnement. Évaluer. Journal international de recherche en éducation et formation, 1(3), 11-27. Repéré à http://www.sr-hepfr.ch/pfcoen/pfcoen/publications files/e jiref 2 gremion coen.pdf

Gremion, C. et Maubant, P. (2017). Évaluation et étayage de l'analyse de pratique. Phronesis, 6(4), 1-10. http://dx.doi.org/10.7202/1043976ar

Hadji, C. (2012). Faut-il avoir peur de l'évaluation? Bruxelles : De Boeck. http://dx.doi.org/10.3917/dbu.hadji.2012.01

Jorro, A. (2004). Réflexivité et auto-évaluation dans les pratiques enseignantes. Mesure et évaluation en éducation, 27(2), 33-47. Repéré à https://halshs.archives-ouvertes.fr/halshs-00112337

Jorro, A. (2016). De l'évaluation à la reconnaissance professionnelle en formation. Revue française de pédagogie, (190), 41-50. http://dx.doi.org/10.4000/rfp.4697

Jorro, A. et Van Nieuwenhoven, C. (2019). Le clair-obscur de l'activité co-évaluative. Dans A. Jorro et N. Droyer (dir.), L'évaluation, levier pour l'enseignement et la formation (1 ${ }^{\text {re }}$ éd., p. 33-44). Bruxelles : De Boeck.

Kaufmann, J.-C. (2001). Ego. Pour une sociologie de l'individu. Paris : Hachette.

Le Boterf, G. (2015). Construire les compétences individuelles et collectives : Agir et réussir avec compétence, les réponses à 100 questions ( $7^{\mathrm{e}}$ éd.). Paris : Eyrolles.

Lemosse, M. (1989). Le «professionnalisme » des enseignants : le point de vue anglais. Recherche et formation, (6), 55-66. http://dx.doi.org/10.3406/refor.1989.982

Lussi Borer, V. et Muller, A. (2014). Exploiter le potentiel des processus de renormalisation en formation à l'enseignement. Activités, 11(2). http://dx.doi.org/10.4000/activites.967

Maubant, P. (2013). Apprendre en situations : un analyseur de la professionnalisation dans les métiers adressés à autrui. Québec, QC : Presses de l'Université du Québec.

Maulini, O. et Vincent, V. (2014). Du travail réel aux pratiques souhaitées : rapport au savoir et rapport au devoir en formation des enseignants. Dans L. Paquay, P. Perrenoud, M. Altet, R. Étienne et J. Desjardins (dir.), Travail réel des enseignants et formation: Quelle référence au travail des enseignants dans les objectifs, les dispositifs et les pratiques? (p. 189204). Louvain-la-Neuve : De Boeck.

Saussez, F. et Allal, L. (2007). Réfléchir sur sa pratique : le rôle de l'autoévaluation? Mesure et évaluation en éducation, 30(1), 97-124.

Schwartz, Y. (2004). L'expérience est-elle formatrice? Les acquis de l'expérience. Éducation permanente, (158), 11-23.

Tardif, M. (2012). Réflexivité et expérience du travail enseignant : repenser le « praticien réflexif » à la lumière des traditions de la pensée réflexive. Dans M. Tardif, C. Borgès et A. Malo (dir.), Le virage réflexif en éducation : où en sommes-nous 30 ans après Schön? (p. 47-71). Bruxelles : De Boeck. http://dx.doi.org/10.3917/dbu.tardi.2012.01.0047

Vaudan Méresse, A. et Gremion, C. (2018). Renormalisation dans un eportfolio. Revue canadienne sur l'avancement des connaissances en enseignement et en apprentissage.

Vial, M. (2006). Accompagner n'est pas guider. Communication présentée à la Conférence aux formateurs de l'École de la Léchère, Bulle. Repéré à http://www.michelvial.com/boite 06 10/2006-Accompagner n est pas guider Conference Suisse.pdf

Vial, M. et Caparros-Mencacci, N. (2007). L'accompagnement professionnel ? Méthode à l'usage des praticiens exerçant une fonction éducative. Bruxelles : De Boeck.

Vial, M. (2012). Se repérer dans les modèles de l'évaluation méthodes dispositifs outils. Bruxelles: De Boeck université. 
Vialle, F. (2005). La construction paradoxale de l'autonomie en formations alternées. Paris : L'Harmattan.

Wittorski, R. (2014). Professionnalisation. Dans A. Jorro (dir.), Dictionnaire des concepts de la professionnalisation (1 ${ }^{\text {re }}$ éd., p. 233-236). Bruxelles : De Boeck.

\section{Pour citer cet article}

Gremion, C. (2019). Accompagner la réflexivité collective, un métier voué à disparaître ? Formation et profession, 27(2), 6-18. http://dx.doi.org/10.18162/fp.2019.490 